\title{
Potential HIV prophylaxis enters Canadian pilot trial
}

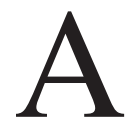

n established treatment for HIV is being studied for realworld use as prophylaxis in two Canadian trials beginning in late March. Truvada (emtricitabine and tenofovir disoproxil fumarate) is already approved in the United States for the prevention of HIV. It is approved in Canada to treat HIV, but its manufacturer, Gilead Sciences Canada, Inc., has "not yet submitted an application" to Health Canada for its use as prophylaxis, stated spokesperson Cara Miller.

As prophylaxis, Truvada, taken once daily and in combination with safer sex practices, reduces the risk of sexually acquired HIV-1 infection, the most common type of HIV.

The Canadian pilot trials for Truvada's use as pre-exposure prophylaxis (PrEP) for high-risk groups are being conducted at the Université de Montréal in Quebec, and St. Michael's Hospital in Toronto, Ontario.

The pilot demonstration project at St. Michael's will follow 50 gay and bisexual men for one year to gauge interest in using PrEP, their adherence, and changes in their sexual and/or risktaking behaviour.

"These are important implementation issues that need to be addressed in order to truly move the field of HIV prevention science forward into 'real life,"” says principal investigator, Dr. Darrell Tan, a clinician-scientist in infectious diseases at the hospital.

Truvada is already being used offlabel as a prophylaxis, but not on a large scale, according to Tan. "Most people are waiting for 'real world' data to inform a roll-out."

$\mathrm{He}$ adds that PrEP is "a really important tool in our toolbox."

"Even with emphasizing behaviour change and simple things like condom usage, we see HIV rates in Canada aren't going away. In particular, the rates in gay, bisexual and other MSM [men-who-have-sex-with-men] populations are much the same, year after year after year."

Dr. Ann Stewart, medical director of Casey House, a facility in Toronto that provides treatment, support and palliative care for patients with HIV/AIDS, agrees. "We've had years of education about using condoms and HIV, but clearly that isn't working. So we need to try something new."

If Gilead submits an application to Health Canada to approve Truvada as a prophylactic agent, it could prove very useful for patients at high risk of contracting HIV, including sex workers, the homeless and MSM.

Truvada will help prevent HIV, but it won't address the underlying issues many high-risk people face, cautions Dr. Sean Rourke, scientific and executive director of the Ontario HIV Treatment Network.

"For prevention, you just can't do it without looking at the other things that put people at risk," says Rourke. "People need support, they need housing, they need to learn new skills, be in better environments, and not experience discrimination." — Jack Lawson, CMAJ

\section{CMAJ 2014. DOI:10.1503/cmaj.109-4750}

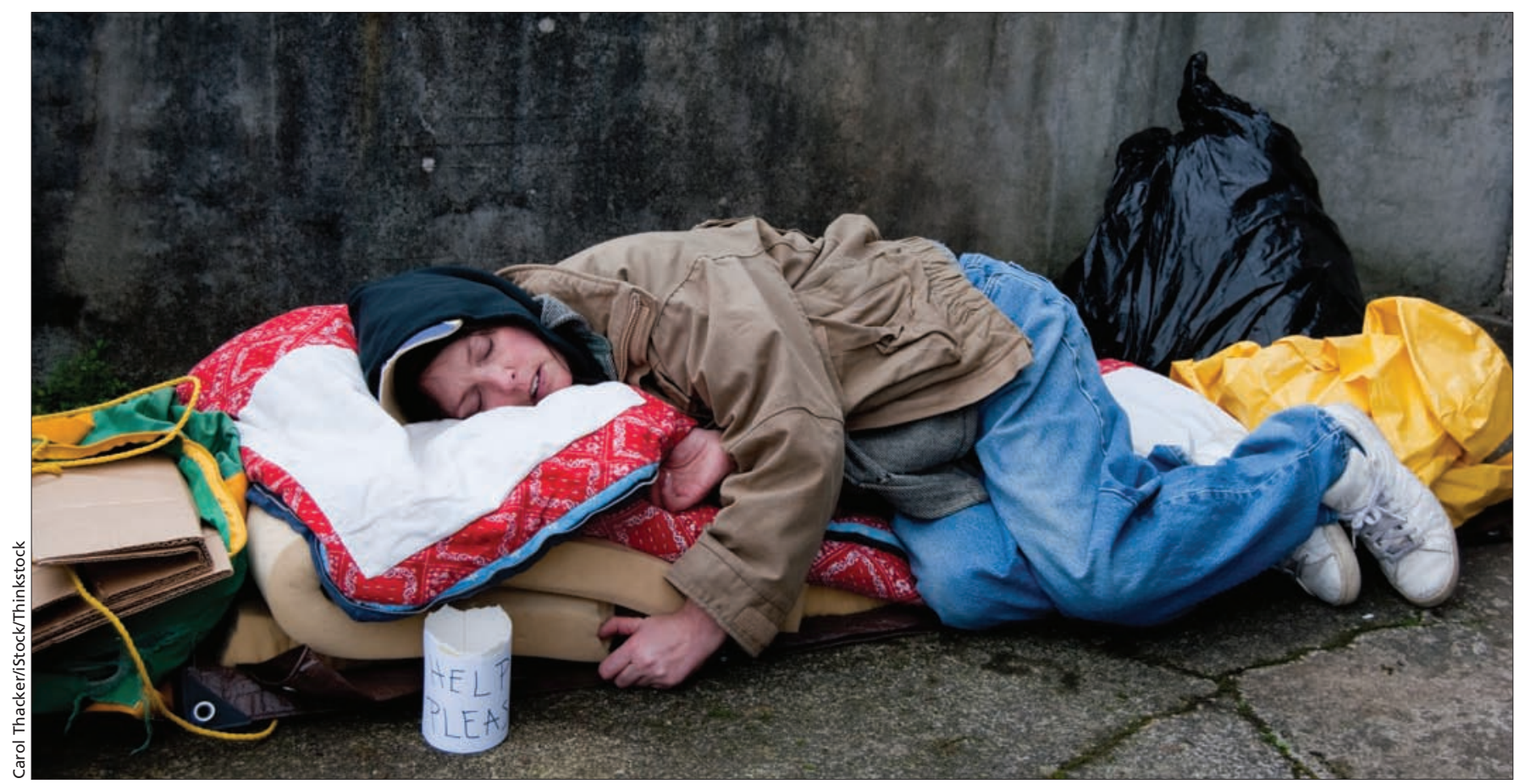

Homeless people, as well as others at high risk of contracting HIV, could potentially benefit from Truvada. 